\title{
Unsteady state thermoelastic problem in a circular annular fin due to internal heat source
}

\author{
C.M. Jadhav ${ }^{1}$ and B.R.Ahirrao ${ }^{2}$ \\ ${ }^{I}$ (Department of Mathematics, Dadasaheb Rawal College Dondaicha, NMU Jalgaon University, Jalgaon \\ [M.S.], INDIA \\ ${ }^{2}$ (Departments of Mathematics, Z.B.Patil College, Dhule, NMU Jalgaon University, Jalgaon INDIA)
}

\begin{abstract}
This paper is concerned with the transient unsteady state thermoelastic problem of thin circular annular fin due to heat source. The transient heat conduction equation is solved by Marchi Zgrablich and Laplace transform with radiation boundary condition. The solution of the problem in the form of infinite series of Bessel function. To determine temperature distribution for heating process and their stresses .Numerical calculations are carried out by using mathematica software.
\end{abstract}

Keywords - Transient distribution, integral transform, heat source, circular annular fin, and transient heating

\section{INTRODUCTION}

The circular annular fin is found in many field of thermal engineering such as air conditioning, heat exchangers microelectronics. Circular annular fin are used mostly in heat exchange devices to increase the heat transfer rate from a heat source for a given temperature difference or to decrease the temperature difference between the heat source and heat sink for a given heat flow rate. Several solution to the problem of one dimensional steady state condition within an annular fin of constant thickness have been presented [2],[5],[6]] and [8].The typical problem of transient thermal stresses in one dimensional steady state condition within an annular fin of constant annular fin have been investigated by $\mathrm{Wu}[9]$.

The present paper attempt to generalize the one dimension problem considered by $\mathrm{Wu}$ [9] and obtains the exact solution of two dimensional transient heat equation problem with radiation boundary condition subjected to internal heat source.

\section{Formulation Of The Problem}

l, where

We consider circular annular fin Fig. 1 occupying the space $D=\left\{(x, y, z) \in R^{3}: a \leq r \leq b, 0 \leq z \leq\right.$

$r=\sqrt{x^{2}+y^{2}}$ The material of fin is isotropic homogeneous and all properties are assumed to be constant. The governing equations and boundary condition for the stress field [12] are

A nonzero stress strain-displacement equation $\varepsilon_{r}=\frac{\partial u}{\partial r}, \varepsilon_{\varphi}=\frac{u}{r}$

A single equilibrium equation $\frac{d \sigma_{r}}{d r}+\frac{\sigma_{r}-\sigma_{\varphi}}{r}=0$

Two equation of stress strain equation relation

$\sigma_{r}=\frac{E}{1-v^{2}}\left[\varepsilon_{r}-v \varepsilon_{\varphi}-(1+v) \alpha T\right]$

$\sigma_{\varphi}=\frac{E}{1-v^{2}}\left[\varepsilon_{r}-v \varepsilon_{\varphi}+(1+v) \alpha T\right]$

And boundary condition

$\sigma_{r}=0$ at $r=a, \sigma_{\varphi}=0$ at $r=b$

Combining equation (2.1)-(2.4), integrating twice the restive $r$ and applying the boundary condition, one obtain the stress strain displacement relation as

$$
\begin{aligned}
& \sigma_{r}=\frac{-\alpha E}{r^{2}} \int_{a}^{r}\left(T-T_{\infty}\right) \eta d \eta+\frac{\alpha E}{b^{2}-a^{2}}\left(1-\frac{a^{2}}{r^{2}}\right) \int_{a}^{b}\left(T-T_{\infty}\right) \eta d \eta \\
& \sigma_{\varphi}=-\alpha E\left(T-T_{\infty}\right)+\frac{\alpha E}{r^{2}} \int_{a}^{r}\left(T-T_{\infty}\right) \eta d \eta+\frac{\alpha E}{b^{2}-a^{2}}\left(1+\frac{a^{2}}{r^{2}}\right) \int_{a}^{b}\left(T-T_{\infty}\right) \eta d \eta
\end{aligned}
$$

Introduce dimensional quantities $\theta, \xi, \tau, R, S_{r}, S_{\varphi}$ in (2.6), (2.7)

$S_{r}=\frac{-1}{\xi^{2}} \int_{1}^{\xi} \theta \xi d \xi+\frac{1}{\xi^{2}} \frac{\xi^{2}-1}{R^{2}-1} \int_{1}^{R} \theta \xi d \xi$

$S_{\varphi}=-\theta+\frac{1}{\xi^{2}} \int_{1}^{\xi} \theta \xi d \xi+\frac{1}{\xi^{2}} \frac{\xi^{2}+1}{R^{2}-1} \int_{1}^{R} \theta \xi d \xi$

Unsteady -state conduction with an isotropic, circular annular fin with internal heat source must satisfy two dimensional equation, which in cylindrical coordinate can be obtained by substituting the radial and tangential stresses in to stress equilibrium equation, lead as $a \leq r \leq b, 0 \leq z \leq l, t>0$ 
$k\left(\frac{\partial^{2} T}{\partial r^{2}}+\frac{1}{r} \frac{\partial T}{\partial r}+\frac{\partial^{2} T}{\partial z^{2}}\right)-\frac{2 h}{l}\left(T-T_{\infty}\right)+\psi(r, z, t, T)=\rho c \frac{\partial T}{\partial t} \quad, a \leq r \leq b, 0 \leq z \leq l, t>0$

Substitute dimensional parameters

$\theta=\frac{k\left(T-T_{\infty}\right)}{\rho b a}, \xi=\frac{r}{a}, \zeta=\frac{z}{a}, L=\frac{l}{a}, \tau=\frac{k t}{\rho c a}, R=\frac{b}{a}, N^{2}=2 h a^{2} / k l$

$\psi(r, z, t, T)=\Phi(r, z, t)+\varepsilon(t) T(r, z, t)$

$\chi(\xi, \zeta \tau)=\Phi(r, z, t) e^{-\int_{0}^{t} \varepsilon(y) d y}$

For sake of brevity, we consider $\chi(\mathrm{r}, \mathrm{z}, \mathrm{t})=\frac{\delta\left(\mathrm{r}-\mathrm{r}_{0}\right) \delta\left(\mathrm{z}-\mathrm{z}_{0}\right)}{2 \pi \mathrm{r}_{0}} \mathrm{e}^{-\omega \mathrm{t}}$ one obtain

$\frac{\partial^{2} \theta}{\partial \xi^{2}}+\frac{1}{\xi} \frac{\partial \theta}{\partial r}+\frac{\partial^{2} \theta}{\partial \zeta^{2}}-N^{2}+\chi(\xi, \zeta \tau)=\frac{\partial \theta}{\partial t}, 1 \leq \xi \leq R, 0 \leq \zeta \leq L, \tau>0$

Subject to initial and boundary conditions

$M_{\tau}(\theta, 1,0,0)=0$, for $1 \leq \xi \leq R, 0 \leq \zeta \leq L, \tau=0(2.12)$

$M_{\xi}\left(\theta, 1, \mathrm{k}_{1}, 1\right)=0, M_{\xi}\left(\theta, 1, \mathrm{k}_{2}, R\right)=\mathrm{F}_{1}(\zeta, \tau)$, for $, 0 \leq \zeta \leq L, \tau>0$

$M_{\zeta}(\theta, 1,0,0)=0, M_{\zeta}(\theta, 1,0, L)=\mathrm{f}(\xi, \tau)$,for $1 \leq \xi \leq R, \tau>0$

Being $M_{\vartheta}(f, \bar{k}, \overline{\bar{k}}, \$)=(\bar{k} f+\overline{\bar{k}} \dot{f})_{\vartheta=\$}$

Where the dot denotes differentiation with respective to $\vartheta, \mathrm{k}_{1}, \mathrm{k}_{2}$ are radiation constant on the curved surface of the annular disc thus equations (2.1) to (2.15)constitute the mathematical formulation of the heating problem under consideration .

\section{Solution Of The Problem}

Applying March Zgrablich integral transform to the (2.11), (2.12) and (2.14) using (2.13) and taking Laplace transform, one obtain

$\bar{\theta}^{*}(n, \zeta, s)=\left[\bar{f}^{*}(n, s)-(P I)_{\zeta=L}\right] \frac{\operatorname{Sinh} p \zeta}{\operatorname{Sinh} p L}+(P I)_{\zeta=0} \frac{\operatorname{Sinh} p(\zeta-L)}{\operatorname{Sinh} p L}$

where $p^{2}=\mu_{n}^{2}+N^{2}+\frac{s}{k}, P I=\frac{k \bar{\Psi}^{*}}{D^{2-} p^{2}}$

Applying inverse of March Zgrablich and Laplace integral transform to (3.1) we obtain

$\theta(\zeta, \zeta \tau)=$

$-\frac{2 k \pi}{L^{2}} \sum_{n, m=1}^{\infty} \frac{S_{0}\left(\mathrm{k}_{1}, \mathrm{k}_{2}, \mu_{\mathrm{m}} \xi\right)}{C_{m}}(-1)^{n} n \int_{0}^{\tau} \operatorname{Sinh}\left(\lambda_{n} \zeta\right)\left[\bar{f}(n, s)-[P I]_{\zeta=L}^{\prime}\right]+\operatorname{Sinh} \lambda_{n}(\zeta-$

$L)[P I]_{\zeta=0}^{\prime} e^{-\left(\mu_{m}{ }^{2}+N^{2}+\lambda_{n}{ }^{2}\right)\left(\tau-\tau^{\prime}\right)} d \tau^{\prime}$

where $\mu_{\mathrm{m}}$ are the positive roots of $J_{0}\left(\mathrm{k}_{1}, \mu\right) Y_{0}\left(\mathrm{k}_{2}, \mu R\right)-J_{0}\left(\mathrm{k}_{2}, \mu \mathrm{R}\right) Y_{0}\left(\mathrm{k}_{1}, \mu\right)=0$ and dash indicate inverse Laplace transform, $\lambda_{n}=\frac{\pi n}{L}$

IV. Determination Thermal Stresses

$S_{r}=$

Using (3.2)in (2.8),(2.9) we obtain the radical and tangential stresses are

$\frac{2 k \pi}{L^{2}} \frac{1}{\xi^{2}} \int_{1}^{\xi} \sum_{n, m=1}^{\infty} \frac{(-1)^{n} n \xi S_{0}\left(\mathrm{k}_{1}, \mathrm{k}_{2}, \mu_{\mathrm{m}} \xi\right)}{C_{m}} \int_{0}^{\tau} \operatorname{Sinh}\left(\lambda_{n} \zeta\right)\left[\bar{f}(n, s)-[P I]_{\zeta=L}^{\prime}\right]+\operatorname{Sinh} \lambda_{n}(\zeta-$

L) $P I^{\prime} \zeta=0 e-\mu m 2+N 2+\lambda n 2 \quad \tau-\tau^{\prime} d \tau^{\prime} d \xi-2 k \pi L 21 \xi 2 \xi 2-1 R 2-11 R n, m=100$

$-1 n n \xi S O \mathrm{k} 1, \mathrm{k} 2, \mu \mathrm{m} \xi \operatorname{CmO} 0 \operatorname{Sinh}(\lambda n \zeta) \quad f(n, S)-P I^{\prime} \zeta=L+\operatorname{Sinh} \lambda n(\zeta-L) P I^{\prime} \zeta=0 e-\mu m 2+N 2+\lambda n 2 \quad \tau-\tau^{\prime} d \tau^{\prime} d \xi$ $(4.1)$

$S_{\varphi}=$

$\frac{2 k \pi}{L^{2}} \sum_{n, m=1}^{\infty} \frac{(-1)^{n} n S_{0}\left(\mathrm{k}_{1}, \mathrm{k}_{2}, \mu_{\mathrm{m}} \xi\right)}{C_{m}} \int_{0}^{\tau} \operatorname{Sinh}\left(\lambda_{n} \zeta\right)\left[\bar{f}(n, s)-[P I]_{\zeta=L}^{\prime}\right]+\operatorname{Sinh} \lambda_{n}(\zeta-$

$L)[P I]_{\zeta=0}^{\prime} e^{-\left(\mu_{m}{ }^{2}+N^{2}+\lambda_{n}{ }^{2}\right)\left(\tau-\tau^{\prime}\right)} d \tau^{\prime}-\frac{2 k \pi}{L^{2}} \frac{1}{\xi^{2}} \int_{1}^{\xi} \sum_{n, m=1}^{\infty} \frac{(-1)^{n} n \xi S_{0}\left(\mathrm{k}_{1}, \mathrm{k}_{2}, \mu_{\mathrm{m}} \xi\right)}{C_{m}} \int_{0}^{\tau} \operatorname{Sinh}\left(\lambda_{n} \zeta\right)\left[\bar{f}(n, s)-[P I]_{\zeta=L}^{\prime}\right]+$ $\operatorname{Sinh} \lambda n(\zeta-L) P I^{\prime} \zeta=0 e-\mu m 2+N 2+\lambda n 2 \quad \tau-\tau^{\prime} d \tau^{\prime} d \xi-2 k \pi L 21 \xi 2 \xi 2+1 R 2-11 R n, m=1 \infty$ $-1 n n \xi S O \mathrm{k} 1, \mathrm{k} 2, \mu \mathrm{m} \xi C \mathrm{Cm} 0 \tau \operatorname{Sinh}(\lambda n \zeta) \quad f(n, s)-P I^{\prime} \zeta=L+\operatorname{Sinh} \lambda n(\zeta-L) P I^{\prime} \zeta=0 e-\mu m 2+N 2+\lambda n 2 \tau-\tau^{\prime} d \tau^{\prime} d \xi$

\section{Special Case}

Setting $\quad \mathrm{f}(\xi, \tau)=0, \chi(\xi, \zeta, \tau)=\frac{\delta\left(\xi-\xi_{0}\right) \mathrm{e}^{(\zeta-\zeta 0)}}{2 \pi \xi_{0}} \mathrm{e}^{-\omega \tau}$

Applying Marchi Zgrablich and Laplace transform to (4.1) one obtains 
$\bar{\Psi}^{*}(\xi, \zeta, \tau)=\frac{S_{0}\left(\mathrm{k}_{1}, \mathrm{k}_{2}, \mu_{\mathrm{m}} \xi_{0}\right)}{2 \pi C_{n}(s+\omega)} \mathrm{e}^{(\zeta-\zeta 0)}$

$[P I]_{\zeta=0}^{\prime}=\frac{S_{0}\left(\mathrm{k}_{1}, \mathrm{k}_{2}, \mu_{\mathrm{m}} \xi_{0}\right)}{2 \pi C_{n}\left(1-\lambda_{n}{ }^{2}\right)} \mathrm{e}^{\zeta} \mathrm{e}^{-\omega \tau}$

$[P I]_{\zeta=L}^{\prime}=\frac{S_{0}\left(\mathrm{k}_{1}, \mathrm{k}_{2}, \mu_{\mathrm{m}} \xi_{0}\right)}{2 \pi C_{n}\left(1-\lambda_{n}{ }^{2}\right)} \mathrm{e}^{(\zeta-\mathrm{L})} \mathrm{e}^{-\omega \tau}$

Substitute the value of (5.3),(5.4) in (3.2),(4.1)and (4.2)one obtains

$\theta(\xi, \zeta \tau)=-\frac{k}{L^{2}} \sum_{n, m=1}^{\infty} \frac{(-1)^{n} n S_{0}\left(\mathrm{k}_{1}, \mathrm{k}_{2}, \mu_{\mathrm{m}} \xi\right) S_{0}\left(\mathrm{k}_{1}, \mathrm{k}_{2}, \mu_{\mathrm{m}} \xi_{0}\right)}{C_{m}\left(1-\lambda_{n}{ }^{2}\right)}\left(\operatorname{Sinh}\left(\lambda_{n} \zeta\right)-\operatorname{Sinh} \lambda_{n}\left(\zeta-\zeta_{0}\right)\right) e^{-\left(\mu_{m}{ }^{2}+N^{2}+\lambda_{n}{ }^{2}+\omega\right) \tau}$

$S_{r}$

$=\frac{k}{L^{2}} \frac{1}{\xi^{2}} \int_{1}^{\xi} \sum_{n, m=1}^{\infty} \frac{(-1)^{n} n \xi S_{0}\left(\mathrm{k}_{1}, \mathrm{k}_{2}, \mu_{\mathrm{m}} \xi\right) S_{0}\left(\mathrm{k}_{1}, \mathrm{k}_{2}, \mu_{\mathrm{m}} \xi_{0}\right)\left(\operatorname{Sinh}\left(\lambda_{n} \zeta\right)-\operatorname{Sinh} \lambda_{n}\left(\zeta-\zeta_{0}\right)\right)}{C_{m}\left(1-\lambda_{n}{ }^{2}\right)} e^{-\left(\mu_{m}{ }^{2}+N^{2}+\lambda_{n}{ }^{2}+\omega\right) \tau} d \xi$

$-\frac{k}{L^{2}} \frac{1}{\xi^{2}} \frac{\xi^{2}-1}{R^{2}-1} \int_{1}^{R} \sum_{n, m=1}^{\infty} \frac{(-1)^{n} n \xi S_{0}\left(\mathrm{k}_{1}, \mathrm{k}_{2}, \mu_{\mathrm{m}} \xi\right) S_{0}\left(\mathrm{k}_{1}, \mathrm{k}_{2}, \mu_{\mathrm{m}} \xi_{0}\right)\left(\operatorname{Sinh}\left(\lambda_{n} \zeta\right)-\operatorname{Sinh} \lambda_{n}\left(\zeta-\zeta_{0}\right)\right)}{C_{m}\left(1-\lambda_{n}{ }^{2}\right)} e^{-\left(\mu_{m}{ }^{2}+N^{2}+\lambda_{n}{ }^{2}+\omega\right) \tau} d \xi$

$S_{\varphi}$

$=\frac{k}{L^{2}} \sum_{n, m=1}^{\infty} \frac{(-1)^{n} n S_{0}\left(\mathrm{k}_{1}, \mathrm{k}_{2}, \mu_{\mathrm{m}} \xi\right) S_{0}\left(\mathrm{k}_{1}, \mathrm{k}_{2}, \mu_{\mathrm{m}} \xi_{0}\right)}{C_{m}\left(1-\lambda_{n}{ }^{2}\right)}\left(\operatorname{Sinh}\left(\lambda_{n} \zeta\right)-\operatorname{Sinh} \lambda_{n}\left(\zeta-\zeta_{0}\right)\right) e^{-\left(\mu_{m}{ }^{2}+N^{2}+\lambda_{n}{ }^{2}+\omega\right) \tau}$

$-\frac{k}{L^{2}} \frac{1}{\xi^{2}} \int_{1}^{\xi} \sum_{n, m=1}^{\infty} \frac{(-1)^{n} n \xi S_{0}\left(\mathrm{k}_{1}, \mathrm{k}_{2}, \mu_{\mathrm{m}} \xi\right) S_{0}\left(\mathrm{k}_{1}, \mathrm{k}_{2}, \mu_{\mathrm{m}} \xi_{0}\right)\left(\operatorname{Sinh}\left(\lambda_{n} \zeta\right)-\operatorname{Sinh} \lambda_{n}\left(\zeta-\zeta_{0}\right)\right)}{C_{m}\left(1-\lambda_{n}{ }^{2}\right)} e^{-\left(\mu_{m}{ }^{2}+N^{2}+\lambda_{n}{ }^{2}+\omega\right) \tau} d \xi$

$-\frac{k}{L^{2}} \frac{1}{\xi^{2}} \frac{\xi^{2}+1}{R^{2}-1} \int_{1}^{R} \sum_{n, m=1}^{\infty} \frac{(-1)^{n} n \xi S_{0}\left(\mathrm{k}_{1}, \mathrm{k}_{2}, \mu_{\mathrm{m}} \xi\right) S_{0}\left(\mathrm{k}_{1}, \mathrm{k}_{2}, \mu_{\mathrm{m}} \xi_{0}\right)\left(\operatorname{Sinh}\left(\lambda_{n} \zeta\right)-\operatorname{Sinh} \lambda_{n}\left(\zeta-\zeta_{0}\right)\right)}{C_{m}\left(1-\lambda_{n}{ }^{2}\right)} e^{-\left(\mu_{m}{ }^{2}+N^{2}+\lambda_{n}{ }^{2}+\omega\right) \tau} d \xi$

The numerical calculation have been carried out for low carbon steel(AISI) with parameter $\mathrm{k}_{1}=\mathrm{k}_{2}=1$, Radius $\mathrm{R}=4, \xi_{0}=1.2, \zeta=0.5 \quad \mathrm{k}=1, \mathrm{~h}=1 \quad \mathrm{~L}=1, \mathrm{~N}=2, \omega=1 \tau=1 \quad \xi=1.5 \quad$ and $\mu_{\mathrm{m}}=1.10821,2.13634,3.17041,4.21067,5.2536,6.29793 .7 .34305,8.38869,9.43467,21.9954$ are the positive root of transcendental equation of $J_{0}\left(\mathrm{k}_{1}, \mu\right) Y_{0}\left(\mathrm{k}_{2}, \mu R\right)-J_{0}\left(\mathrm{k}_{2}, \mu \mathrm{R}\right) Y_{0}\left(\mathrm{k}_{1}, \mu\right)=0 \quad$ and , $\lambda_{n}=3.142,6.284,9.426,12.568,15.71,18.853,21.994,25.136,28.278,31.42$ are the positive root of transcendental equation of $\lambda_{n}=\frac{\pi n}{L}$

$\theta(\xi, \zeta \tau)=-\sum_{n, m=1}^{\infty} \frac{(-1)^{n} n S_{0}\left(\mathrm{k}_{1}, \mathrm{k}_{2}, \mu_{\mathrm{m}} \xi\right) S_{0}\left(\mathrm{k}_{1}, \mathrm{k}_{2}, \mu_{\mathrm{m}} 1.2\right)}{C_{m}\left(1-\pi n^{2}\right)}\left(\operatorname{Sinh}\left(\lambda_{n} \zeta\right)-\operatorname{Sinh} \lambda_{n}\left(\zeta-\zeta_{0}\right)\right) e^{-\left(\mu_{m}{ }^{2}+5+n \pi^{2}\right)}$

$S_{r}=$

$\frac{1}{2.25} \int_{1}^{1.5} \sum_{n, m=1}^{\infty} \frac{(-1)^{n} \xi n S_{0}\left(\mathrm{k}_{1}, \mathrm{k}_{2}, \mu_{\mathrm{m}} \xi\right) S_{0}\left(0.8,1, \mu_{\mathrm{m}} 1.2\right)\left(\operatorname{Sinh}\left(\lambda_{n} \zeta\right)-\operatorname{Sinh} \lambda_{n}\left(\zeta-\zeta_{0}\right)\right)}{C_{m}\left(1-\pi n^{2}\right)} e^{-\left(\mu_{m}^{2}+5+n \pi^{2}\right)} d \xi-$

$\frac{1}{2.7} \int_{1}^{2} \sum_{n, m=1}^{\infty} \frac{(-1)^{n} n \xi S_{0}\left(\mathrm{k}_{1}, \mathrm{k}_{2}, \mu_{\mathrm{m}} \xi\right) S_{0}\left(0.8,1, \mu_{\mathrm{m}} 1.2\right)\left(\operatorname{Sinh}\left(\lambda_{n} \zeta\right)-\operatorname{Sinh} \lambda_{n}\left(\zeta-\zeta_{0}\right)\right)}{C_{m}\left(1-\pi n^{2}\right)} e^{-\left(\mu_{m}{ }^{2}+5+n \pi^{2}\right)} d \xi$

$S_{\varphi}=$

$\sum_{n, m=1}^{\infty} \frac{(-1)^{n} n S_{0}\left(\mathrm{k}_{1}, \mathrm{k}_{2}, \mu_{\mathrm{m}} \xi\right) S_{0}\left(\mathrm{k}_{1}, \mathrm{k}_{2}, \mu_{\mathrm{m}} 1.2\right)}{C_{m}\left(1-\pi n^{2}\right)}\left(\operatorname{Sinh}\left(\lambda_{n} \zeta\right)-\operatorname{Sinh} \lambda_{n}\left(\zeta-\zeta_{0}\right)\right) e^{-\left(\mu_{m}{ }^{2}+5+n \pi^{2}\right)}-$

$\frac{1}{2.25} \int_{1}^{1.5} \sum_{n, m=1}^{\infty} \frac{(-1)^{n} n \xi S_{0}\left(\mathrm{k}_{1}, \mathrm{k}_{2}, \mu_{\mathrm{m}} \xi\right) S_{0}\left(\mathrm{k}_{1}, \mathrm{k}_{2}, \mu_{\mathrm{m}} 1.2\right)}{C_{m}\left(1-\pi n^{2}\right)}\left(\operatorname{Sinh}\left(\lambda_{n} \zeta\right)-\operatorname{Sinh} \lambda_{n}\left(\zeta-\zeta_{0}\right)\right) e^{-\left(\mu_{m}^{2}+5+n \pi^{2}\right)} d \xi-$

$\frac{13}{13.5} \int_{1}^{2} \sum_{n, m=1}^{\infty} \frac{(-1)^{n} n \xi S_{0}\left(\mathrm{k}_{1}, \mathrm{k}_{2}, \mu_{m} \xi\right) S_{0}\left(\mathrm{k}_{1}, \mathrm{k}_{2}, \mu_{\mathrm{m}} 1.2\right)}{C_{m}\left(1-\pi n^{2}\right)}\left(\operatorname{Sinh}\left(\lambda_{n} \zeta\right)-\operatorname{Sinh} \lambda_{n}\left(\zeta-\zeta_{0}\right)\right) e^{-\left(\mu_{m}{ }^{2}+5+n \pi^{2}\right)} d \xi$ 


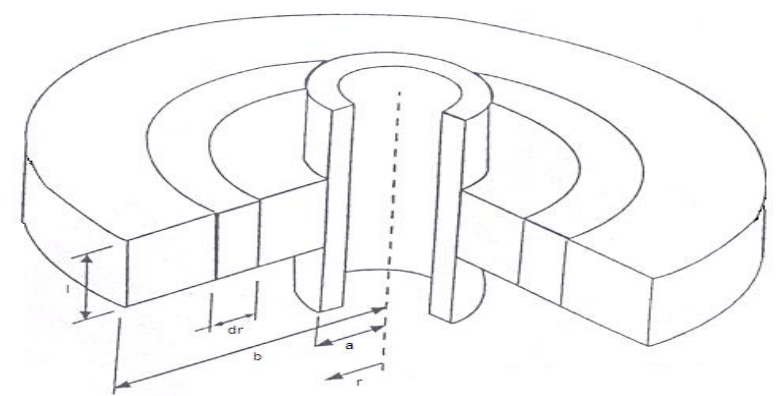

Figure 1: Characteristics of the annular fin

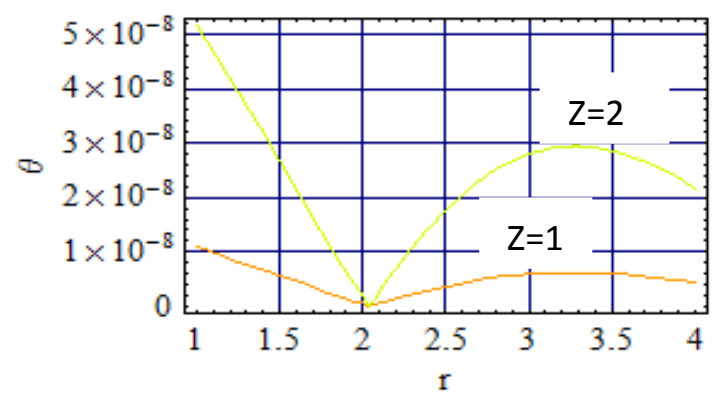

Fig.2Temperature verses $r$ with different value of $\mathrm{z}$ at $\mathrm{t}=1$

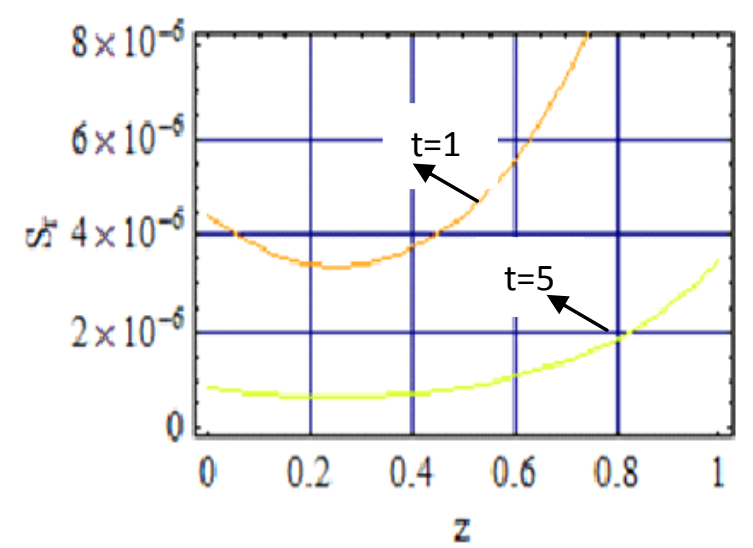

Fig.3 Thermal Stress $S_{r}$ verses $\mathrm{r}$ with different time

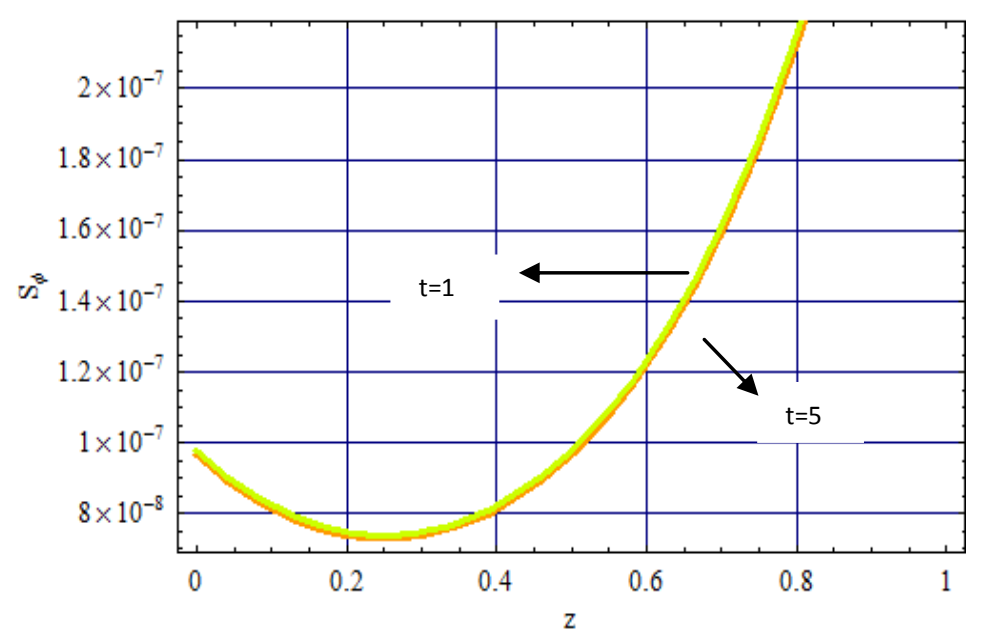

Fig.4Thermal stress $S_{\varphi}$ verses $\mathrm{r}$ with different time 


\section{Conclusion}

In this paper, we generalized the idea proposed by $\mathrm{Wu}$ et.al(1997) for two dimensional non homogeneous radiation boundary value problem of circular annular fin with heat source and temperature distribution Displacement and stress function for annular fin have been obtained we developed the analysis for temperature field for heating processes by using March Zgrablich and Laplace transform technique with boundary condition of radiation type. the series solution is converges since the thickness of annular fin is very small also any particular case of special interest may be derived by assigning suitable value of the parameter and function in the series expansion. The result can be applied to the design of useful structures or machines in engineering applications.

\section{Acknowledgements}

The authors express their sincere thanks to Dr. N. W. Khobragade for their expert comments.

\section{References}

[1] Boley, B.A. and Weiner, J.H., Theory of thermal stresses, Johan Wiley and Sons, New York, (1960)

[2] Carrier,W.H. and Aderson ,S.W., The resistance of heat flow through finned tubing, heating piping and Air conditioning ,Vol.10 ,1944,pp.304-320.

[3] Carslaw, S.H., and Jaeger J.C., Conduction of heat in solid, second edition, oxford University Press, New york (1959)

[4] Crandall S.H., DahlN.C. and Lardner T.J. ,An introduction to the mechanics of solids ,McGraw-Hill, New york(1978)

[5] Gardner K.A., Efficiency of extended surfaces, transaction of American society of mechanical engineers, vol.67, 1945, pp621-631.

[6] Harper, D.R., and Brown W.B. , Mathematica; equation for Heat conduction in Fins of air -cooled Engines .NACA Report No.158,(1922).

[7] Marchi E. and Zgrablich, J., Heat conduction in hollow cylinder with radiation processing Edinburg math .Soc. Vol.14 1964(Series 11) part 2pp.159-164.

[8] Murray W.M., Heat dissipation through an annular disc or fin of uniform thickness, Journal of applied mechanics Vol.5Transaction of the American society of mechanical engineers VOl.60, 1938, pA-78

[9] Wu, S.S., Analysis of transient thermal stresses in annular fin, Journal of thermal stresses, vol 20, 1997,pp591-615.

[10] Deshmukh K.C. and Kedar D.G., Estimation of temperature distribution and thermal stresses in a thick circular plate ,Afre.J.Math Comp.Sci.Res.4 (B) pp389-395, 2011

[11] Shang Sheng W., analysis on transient thermal stresses in an annular fin J. Thermal stresses 20,1999,pp.591-615.

[12] Warde R.W. Deshmukh K.C., Mathematical modeling on transient thermal stresses in an annular fin Acta ciencia ,XXIX M(3),2003,pp507-516.

[13] Navneet Kumar Lamba, Warbhe M.S. and Khobragade N.W., Temperature dependant thermal stress determination of isotropic circular annular fin ,Affric J Math Sci. Cop .Res.Vol.5,pp94-11)

\section{Appendix}

The finite Marchi-Zgrablich integral transform of order $p$ is defined as

$\bar{f}_{p}(n)=\int_{a}^{b} r f(r) S_{p}\left(\mathrm{k}_{1}, \mathrm{k}_{2}, \mu_{\mathrm{n}} r\right) d r$

And inverse Marchi-Zgrablich integral transform as

$f(r)=\sum_{n=1}^{\infty} \frac{\bar{f}_{p}(n) S_{p}\left(\mathrm{k}_{1}, \mathrm{k}_{2}, \mu_{\mathrm{n}} r\right)}{C_{n}}$

Where

$S_{p}\left(\mathrm{k}_{1}, \mathrm{k}_{2}, \mu_{\mathrm{n}} r\right)=J_{p}\left(\mu_{\mathrm{n}} r\right)\left\{Y_{p}\left(\mathrm{k}_{1}, \mu_{\mathrm{n}} a\right)+Y_{p}\left(\mathrm{k}_{2}, \mu_{\mathrm{n}} b\right)\right\}-Y_{p}\left(\mu_{\mathrm{n}} r\right)\left\{J_{p}\left(\mathrm{k}_{1}, \mu_{\mathrm{n}} a\right)+J_{p}\left(\mathrm{k}_{2}, \mu_{\mathrm{n}} b\right)\right\}$

$C_{n}=\int_{a}^{b}\left[r S_{p}\left(\mathrm{k}_{1}, \mathrm{k}_{2}, \mu_{\mathrm{n}} r\right)\right]^{2} d r$

The eigenvalue $\mu_{\mathrm{n}}$ are the positive roots of the equation $J_{p}\left(\mathrm{k}_{1}, \mu_{\mathrm{n}} a\right) Y_{p}\left(\mathrm{k}_{2}, \mu_{\mathrm{n}} b\right)-J_{p}\left(\mathrm{k}_{2}, \mu_{\mathrm{n}} b\right) Y_{p}\left(\mathrm{k}_{1}, \mu_{\mathrm{n}} a\right)=0$

An operational property is given by

$\int_{a}^{b}\left[\frac{\partial^{2} f}{\partial x^{2}}+\frac{1}{x} \frac{\partial f}{\partial x}+\frac{p^{2} f}{x^{2}}\right] S_{p}\left(\mathrm{k}_{1}, \mathrm{k}_{2}, \mu_{\mathrm{n}} r x\right)$

$=\frac{b}{\mathrm{k}_{2}} S_{p}\left(\mathrm{k}_{1}, \mathrm{k}_{2}, \mu_{\mathrm{n}} b\right)\left[f+\mathrm{k}_{2} \frac{\partial f}{\partial r}\right]_{r=b}$

$-\frac{a}{\mathrm{k}_{1}} S_{p}\left(\mathrm{k}_{1}, \mathrm{k}_{2}, \mu_{\mathrm{n}} a\right)\left[f+\mathrm{k}_{1} \frac{\partial f}{\partial r}\right]_{r=a}-\mu_{\mathrm{n}}^{2} \bar{f}_{p}(n) \quad$ and $J_{p}\left(\mu_{\mathrm{n}} r\right)$ and $Y_{p}\left(\mu_{\mathrm{n}} r\right)$ are the Bessel function of first and second kind respectively.

Nomenclature

a, b: Inner and outer radii of the fin

$\mathrm{c}_{1}, \mathrm{c}_{2}$ : Constants

h: Heat transfer coefficient

$\mathrm{N}$ : Dimensionless parameter

$\mathrm{q}_{\mathrm{b}}$ : Heat flux from the base of the fin

$S_{r}$ : Dimensionless radial

$\mathrm{T}$ : Temperature of the fin

U: Radial displacement

$\varepsilon_{r}, \varepsilon_{\varphi}:$ Radial and tangential strains

$\sigma_{r}, \sigma_{\theta}$ : Radial and tangential stresses

$\mathrm{r}, \varphi$ :Polar coordinates

c: Specific heat of material of the fin

E: Young's modulus of material of the fin

$\mathrm{k}$ : Thermal conductivity of material of the fin

t: Time

R: Dimensionless outer radius,

$S_{\varphi}$ : Dimensionless tangential stresses

$T_{\infty}$ : Ambient temperature

1: Thickness of the fin

$\theta$ : Dimensionless temperature of the fin

$\tau$ : Dimensionless time

L: Dimensionless thickness 